\title{
LES RELATIONS ECONOMIQUES ET COMMERCIALES ENTRE LA RDC ET LA CHINE : CADRE JURIDIQUE ET INCIDENCES SUR LES DROITS ECONOMIQUES ET SOCIAUX DES CONGOLAIS
}

\author{
Par Willy Wogbo Koberelo ${ }^{1}$
}

\section{Résumé}

Cet article examine le cadre juridique des relations économiques et commerciales entre la RDC et la Chine appréhendé sous deux volets. Le premier qui porte sur les rapports interétatiques tels que réglementés notamment par les principes et règles de droit international et le second sur les rapports transnationaux marqués singulièrement par la convention de collaboration du 22 avril 2008 relative au développement d'un projet minier et d'un projet d'infrastructures en RDC. Bien que cette convention ait permis la réalisation de plusieurs infrastructures, elle n'a pas encore pleinement répondu aux attentes des populations congolaises. Pour que la convention serve d'outil de promotion et de protection des droits économiques et sociaux des populations congolaises, l'Etat congolais devra mettre en place un cadre propice à l'émergence des principes de bonne gouvernance, de gestion transparente qui permettront d'affecter, comme il se doit, les financements issus de l'exploitation des ressources naturelles à l'amélioration des conditions de vie des populations congolaises.

\section{INTRODUCTION}

L'expansion universelle de l'idéologie du libéralisme économique, aidée par l'élimination progressive des obstacles à la liberté de circulation des marchandises, des personnes et des capitaux, instaure une mondialisation de l'économie ${ }^{2}$ qui conduit les Etats à établir des relations économiques et commerciales en vue de générer un développement économique indispensable à l'amélioration des conditions de vie de leurs populations. Ne faisant pas exception à la règle, la Chine et la République Démocratique du Congo(RDC) ont convenu de développer leurs relations en facilitant, le 22 avril 2008, la signature d'un accord entre la RDC et un groupement d'entreprises chinoises.

Loin d'être un acte isolé, cet accord s'inscrit dans le cadre de la politique économique de la Chine, qui, en vue de pourvoir à ses besoins en matières premières, indispensables à la poursuite de sa croissance économique, a conclu avec plusieurs Etats africains, des accords

1 Assistant à la Faculté de Droit de l’Université de Kinshasa, willywogbo@hotmail.fr.

2 Voir Jean Charpentier, Institutions internationales, Paris, 2002, p.15. 
lui permettant d'investir dans le domaine des ressources naturelles et de les exploiter en échange d'infrastructures nécessaires au développement des partenaires africains avec lesquels elle a créé le Forum Chine-Afrique. ${ }^{3}$

Bien que la RDC, comme d'autres pays africains, soit partie prenante à ce forum, l'exploitation des ressources naturelles faisant difficilement l'objet d'un accord multilatéral ${ }^{4}$, la matérialisation de cette coopération sino-congolaise passait nécessairement par un accord apte à reproduire les conditions dans lesquelles la République Démocratique du Congo consentait à l'exploitation de ses ressources naturelles en contrepartie du financement de la construction d'infrastructures nécessaires à son développement. La convention minière du 22 avril 2008 signée par l'Etat congolais et un groupement d'entreprises chinoises a ainsi permis la mise en place d'un cadre propice au développement exponentiel des échanges commerciaux entre la RDC et la Chine.

Bien qu'il ressorte de cet accord que l'Etat congolais compte, par les investissements chinois, financer les travaux d'infrastructures nécessaires au développement économique du pays, les résultats mitigés des accords passés avec les partenaires étrangers en vue d'amorcer le développement économique du pays, et qui ont engendré un endettement ${ }^{5}$ contrastant avec l'espoir qu'ils suscitaient, nous amène à nous interroger sur le cadre juridique sécurisant les intérêts des parties en présence (A) et sur les incidences de cette convention sur les droits économiques et sociaux des populations congolaises (B).

\section{A. Cadre juridique organisant les relations économiques et commerciales entre la République Démocratique du Congo et la Chine}

Mis à part les principes et règles de l'Organisation Mondiale du Commerce(OMC) applicables aux rapports interétatiques qu'entretiennent la RDC et la Chine (I), il nous paraît nécessaire de relever l'importance du Forum Chine-Afrique entant que cadre multilatéral définissant le contexte ${ }^{6}$ de l'évolution des rapports transnationaux (II) marqués notamment par la convention minière du 22 avril 2008 (III) qui, bien qu'elle soit en principe, soumise aux

3 Lire Adama Gaye, Le dragon et l'autruche. Essai d'analyse de l'évolution contrastée des relations sino-africaines : sainte ou impie alliance du XXI ${ }^{\text {ème }}$ siècle, Paris, 2006, p. 252.

4 Lire à ce sujet KUMBU ki NGIMBI, Protection légale des investissements privés étrangers en République Démocratique du Congo et coopération eurafricaine, Thèse de Doctorat, Hambourg, RFA, 1998, p. 125.

5 Voir GLOBAL WITNESS, La Chine et le Congo : Des amis dans le besoin, Rapport sur la République démocratique du Congo, Mars 2011, p.22. Selon ce rapport de Global Witness sur la République démocratique du Congo, la dette de l'Etat congolais était évaluée, le $1^{\text {er }}$ juillet 2010, à 12,3 milliards de dollars. La République Démocratique du Congo s'est finalement vu accorder un allègement de sa dette au titre de l'Initiative en faveur des pays pauvres très endettés et de l'Initiative d'allègement de la dette du Fonds monétaire international et de la Banque Mondiale.

6 Lire à ce sujet Rigobert MINANI BIHUZO, « La coopération Chine-Afrique et le débat sur les contrats chinois en RDC in Les Enjeux de la convention minière RDC-CHINE, Kinshasa, 2010, p. 24. 
lois nationales(IV), fait l'objet de nombreuses dérogations au régime de droit commun applicable aux investissements effectués dans le secteur minier congolais(V).

I. Les principes et règles de l'OMC applicables aux rapports interétatiques existant entre la RDC et la Chine

L'OMC trouve son fondement dans des accords internationaux appelés, accords de Marrakech du 15 avril 1994 qui ont été l'aboutissement du cycle de négociations commerciales d'Uruguay (Uruguay Round). ${ }^{7}$ L'Organisation mondiale du Commerce a été instituée le $1^{\text {er }}$ janvier $1995 .^{8}$ Contrairement aux accords du GATT, le processus de l'OMC a été conçu comme un " engagement unique » faisant obligation à tous les membres d'adhérer à tous les accords, bien qu'une période d'ajustement plus longue ait été accordée aux pays en développement. ${ }^{9} \mathrm{Ceci}$, nous permet d'affirmer que l'OMC, ses règles et ses principes, constitue le cadre juridique ou normatif mis sur pied par le système commercial international et réglemente les relations interétatiques en la matière et s'applique aussi aux relations économiques et commerciales entre la RDC et la Chine.

L'OMC a pour objectif de :

- promouvoir les échanges commerciaux sur une base non discriminatoire;

- promouvoir le relèvement des niveaux de vie et des revenus, l'utilisation optimale des ressources mondiales;

- réduire les distorsions commerciales résultant d'une protection insuffisante de la propriété intellectuelle;

- permettre aux pays en développement, surtout les moins avancés, de mieux participer au commerce international, et promouvoir le développement et la protection de l'environnement.

$\mathrm{L}^{\prime} \mathrm{OMC}$ a son siège à Genève ${ }^{10} \mathrm{et}$ a comme règles de base ${ }^{11}:$ la protection des producteurs nationaux que par les droits de douane, la réduction et la consolidation des droits de douane, l'application du principe de la nation la plus favorisée et l'application de la clause du Traitement National pour tous les produits à l'intérieur des frontières des Etats membres.

Ces principes de base s'appliquent aux relations économiques et commerciales entre la République Démocratique du Congo et la Chine étant donné que les deux Etats sont parties aux Traités de Marrakech et par conséquent, membres de celle-ci. L'Organe de règlement

7 Jean Michel KUMBU ki NGIMBI, Législation en matière économique, Kinshasa, 2013, p.149.

8 Voir Jean Charpentier, op.cit, p.131, note 2.

9 Richard BLACKHURST, « Renforcement des capacités en Afrique pour une participation efficace au processus de l'OMC », in L'Afrique et les défis de l'OMC, Paris, 2004, p.13.

10 Richard BLACKHURST, op.cit, p. 4, note 9.

11 Grégoire Bakandeja wa Mpungu, Le droit commercial international : Les Peurs justifiées de l’Afrique face à la mondialisation des marchés, Kinshasa, Paris, Bruxelles, 2001, p. 20. 
des différends qui constitue l'un des grands acquis de l'Uruguay Round ${ }^{12}$ permet à l'OMC d'émettre des mesures de rétorsions à l'encontre des Etats parties ${ }^{13}$.

Au 1er janvier 2008, elle comptait 153 pays membres représentant plus de $98 \%$ du commerce mondial. ${ }^{14}$ La République Démocratique du Congo est membre de l'Organisation Mondiale du Commerce depuis le $1^{\text {er }}$ janvier 1997. La Chine, par contre, a adhéré à l'OMC le 11 décembre $2001^{15}$ après que les engagements d'ouverture des marchés chinois aux importations, comme ceux des partenaires commerciaux aient été concrétisés par l'accord du 17 septembre $2001^{16}$. C'est à ce titre qu'en principe la République Démocratique du Congo peut se prévaloir des principes, règles et mécanismes mis en place par l'OMC dans les rapports interétatiques qu'elle entretient avec ses partenaires.

Toutefois, l'importance et le rôle de ces deux Etats dans l'OMC tout comme dans le système commercial international est d'un contraste criant. La Chine est un atelier, mais un marché aussi. ${ }^{17}$ En 2011, elle occupait le rang de premier exportateur mondial et de deuxième importateur, ses échanges commerciaux ayant été multipliés par cinq, dans les deux sens. ${ }^{18}$

La République Démocratique du Congo comme bien de pays africains a, quant à elle, du mal à trouver sa place dans le système commercial international et à faire face à la concurrence des Etats industrialisés à qui profitent le principe de libre échange et la perméabilité des frontières entretenus par la mondialisation. La faible participation des pays moins développés, comme la RDC, aux travaux de l'OMC fait que ceux-ci n'ont pu véritablement peser dans les négociations. ${ }^{19}$ A titre d'exemple, en 1998, la RDC n'avait qu'un seul délégué résident à Genève. ${ }^{20}$ En plus, les résultats des travaux récents montrent que pour les pays peu développés, il n'existe pas de relation générale entre ouverture commerciale et croissance, et que les coûts liés à la mise en conformité de ces pays avec les obligations de l'OMC peuvent excéder le budget affecté au développement. ${ }^{21}$

12 Grégoire BakandejawaMpungu, op.cit., p.22, note 11.

13 Idem.

14 Jean Michel KUMBU ki NGIMBI, op.cit, Kinshasa, 2013, p.149, note 6.

15 Voir OMC, http://www.wto.org/french/thewto_f/whatis_f/org6_f.htm consulté le 15 octobre 2014.

16 Michel Rainelli,L'Organisation mondiale du commerce, Paris, 2002, p.91.

17 Ses échanges commerciaux ont été multipliés par cinq dans les deux sens. Voir Alain Frachon, « Dix ans de la Chine à l'OMC : bilan » in journal Le Monde, chronique internationale du 29 septembre 2011 disponible sur le site internet : http//www.LeMonde.fr consulté le 07octobre 2014.

18 Idem.

19 Lire Michel Rainelli, op.cit., p.114, note 16.

20 David LUKE, «Renforcement des capacités dans le domaine du commerce pour une meilleure participation de l'Afrique au sein de l'économie mondiale, in L'Afrique et les défis de l'OMC, Paris, 2004, pp 29-47.

21 Lire Michel Rainelli, op.cit, p.114, note 16. 
Conscient de cela, les rapports économiques et commerciaux entre Etats riches et Etats pauvres s'inscrivent dans une logique d'aide au développement. ${ }^{22}$ Une aide au développement que la Chine compte, en échange notamment de l'exploitation des ressources naturelles, offrir aux Etats africains, dont la RDC, dans le cadre du Forum Chine-Afrique.

II. Le forum Chine-Afrique comme champ d'évolution des relations économiques et commerciales entre la République démocratique du Congo et la Chine

Le forum Chine-Afrique lancé en l'an 2000 définit des orientations concrètes pour relancer les relations sino-africaines. ${ }^{23} \mathrm{~A}$ l'heure où les partenaires traditionnels parmi lesquels le FMI et la BIRD, restaient exigeants quand aux conditions d'octroi des moyens financiers pouvant aider les économies de plusieurs Etats africains et pour lesquels les gouvernants acceptaient difficilement les incessants rappels à l'ordre sur le respect des principes démocratiques de bonne gouvernance, de transparence, et les exigences d'un Etat de droit comme conditions de développement économique, la Chine, dans le but d'exploiter les ressources naturelles dont elle a besoin, a conçu sa politique économique et commerciale avec ses partenaires africains sous une approche ne mettant pas en exergue les difficultés qu'avaient certains partenaires africains à respecter les principes de démocratie et de bonne gouvernance.

Néanmoins, cette approche bénéfique pour la Chine jette des doutes sur les retombées économiques des accords entre les entreprises chinoises et la République Démocratique du Congo notamment en ce qui concerne l'amélioration des conditions sociales des populations congolaises quand on sait que les conditions de bonne gouvernance qui avaient fait défaut dans le passé lors des prêts accordés par les institutions de BrettonWoods ne nous semblent pas déjà acquises

Qu'à cela ne tienne, c'est dans le même ordre d'idées que le " Plan d'action d'AddisAbeba », qui a été adopté lors du deuxième forum Chine-Afrique, a défini les idées directrices, les objectifs principaux et les mesures concrètes à prendre pour renforcer la coopération sino-africaine entre 2004 et $2006 .{ }^{24}$ Parmi les principaux termes de ce plan d'action, l'on peut mettre en en exergue le fait que ${ }^{25}$ :

- la Chine et l'Afrique s'engagent à renforcer leurs consultations au sein des Nations Unies, de l'OMC et d'autres organisations internationales,

- sur le plan économique, la Chine et les pays membres du Forum s'engagent à promouvoir leur coopération dans les domaines de l'agriculture, des infrastructures, du commerce, de l'investissement, du tourisme et de l'exploitation des ressources naturelles,

22 Pour plus de détails, lire Jean Charpentier, op.cit, p.133, note 2.

23 Adama Gaye, Le dragon et l'autruche. Essai d'analyse de l'évolution contrastée des relations sinoafricaines : sainte ou impie alliance du XXI ${ }^{\text {ème }}$ siècle, Paris, 2006, p. 252.

24 Lire Adama Gaye,op.cit, p.258, note 23.

25 Adama Gaye,op.cit, p.259,note 23. 
- dans le domaine des infrastructures, la Chine encourage ses entreprises à participer activement aux projets en Afrique et à développer leur coopération dans d'autres secteurs tels que les transports, les télécommunications, l'énergie et l'alimentation en eau et en électricité.

- la Chine et les pays africains membres du Forum s'engagent à promouvoir un commerce équilibré entre eux; elle a décidé de faire bénéficier du tarif zéro une partie des produits en provenance des Etats d'Afrique les moins avancés pour leur accès au marché chinois, et de lancer des négociations bilatérales avec les Etats concernés sur la liste des produits qui seront exemptés de douane, ${ }^{26}$

- la Chine et les pays africains vont promouvoir les investissements dans les deux sens, et les entreprises chinoises sont encouragées par le gouvernement chinois à investir en Afrique, y compris dans la création des coentreprises; les deux parties ont accepté de prendre des mesures de facilitation pour des entreprises chinoises intéressées à investir, ${ }^{27}$

- la Chine et les pays africains vont intensifier leur coopération pour le développement des ressources naturelles et le développement des ressources humaines (éducation, santé, culture).

Ainsi, ces engagements ont été traduits par des actions concrètes entre la RDC et la Chine qui sont illustrées par l'ambassadeur de Chine en RDC en ces termes : « en 2004, le volume des échanges sino-africains atteint 29,47 milliards de dollars, c'est une augmentation de 58,9\% comparé à l'année précédente. Dès le $1^{\mathrm{er}}$ janvier 2005, la Chine attribue l'exonération d'impôt à l'importation de 190 sortes de marchandises venues de 28 pays africains, la RDC est parmi ces 28 pays. La Chine a également annulé la dette des 31 pays africains les moins développés d'une somme de 10,5 milliards de Yuan dont la dette de 150 millions de Yuan de la RDC. Depuis 2003, la Chine a promis de former pendant 3 ans pour les pays africains 10 mille personnes dans les domaines d'administration, de technologie, de gestion, jusqu'à maintenant on a formé 7 mille personnes, et on compte achever l'objectifl'année prochaine. Le plan de service des jeunes volontaires chinois pour l'Afrique a été inauguré. L'Ambassade de Chine à Kinshasa a déjà fait une invitation aux jeunes volontaires chinois de travailler en $R D C »{ }^{28}$

Les conclusions résultant du $2^{\text {ème }}$ forum Chine-Afrique (du 15 au 16 septembre 2003) peuvent être classées en droit des relations internationales, dans la catégorie des actes concertés non conventionnels. En effet, à côté des traités juridiquement obligatoires comme celui instituant l'OMC- la pratique internationale connait de nombreux engage-

26 Ici, nous relevons la prise en considération de l'importance évidente du contexte bilatéral prioritaire.

27 Ces termes expliquent effectivement l'investissement du groupement d'entreprises chinois en RDC qui devra aboutir à la création d'une société mixte.

28 Interview de l'ambassadeur de Chine en RDC publié par le journal Potentiel du $1^{\text {er }}$ octobre 2005 disponible sur http : //cd.china-embassy.org/fra/xw/more/t215218.htm, consulté le 28 octobre 2014. 
ments politiques, déclarations communes, actes finaux des conférences internationales etc., sans valeur juridique, mais dont la portée politique peut être considérable; il s'agit de ce qu'on appelle soft law. ${ }^{29}$

Toutefois, cela n'enlève pas au Forum Chine-Afrique le mérite d'avoir servi de cadre multilatéral décrivant le contexte de la conclusion de la convention de collaboration du 22 avril 2008 entre la RDC et le groupement d'entreprises chinoises.

III. La convention de collaboration du 22 avril 2008 relative au développement d'un projet minier et d'un projet d'infrastructures

Ces dernières années, les relations économiques et commerciales entre la RDC et la Chine se sont intensifiées, non seulement suite aux différents accords interétatiques comme le traité relatif à l'encouragement et à la protection mutuelle des investissements signé entre les gouvernements congolais et chinois à la date du 18 décembre $1997^{30}$ mais aussi et surtout suite à l'accord conclu le 22 avril 2008 entre la RDC et le groupement d'entreprises chinoises $^{31}$ relative au développement d'un projet minier et d'un projet d'infrastructures en RDC.

Nous aborderons dans les lignes qui suivent les objectifs poursuivis, les principales clauses et obligations contenues dans ladite convention.

\section{Objectifs des parties}

La République Démocratique du Congo, représentée par le Ministre en charge des Infrastructures, Travaux publics et Reconstruction d'une part et le groupement d'entreprises chinoises, reconnaissent qu'en signant la présente convention, la République Démocratique du Congo poursuit l'objectif de trouver les ressources financières nécessaires à la réalisation des projets d'infrastructures nationales estimées importantes et urgentes et le groupement d'entreprises Chinoises, celui d'investir dans le domaine des métaux non-ferreux dans les territoires de la République Démocratique du Congo. ${ }^{32}$

\section{Obligations des Parties}

Hormis l'engagement commun de constituer une joint-venture ${ }^{33}$ pour la réalisation du projet minier et d'infrastructures dont une partie des profits servira au remboursement aussi

29 Lire à ce sujet Jean Charpentier, op.cit, Paris, 2002, p.40.

30 Ce traité a été mentionné à l'article 15.3 de la Convention de collaboration du 22 avril 2008.

31 Aux termes de la convention de collaboration du 22 avril 2008, le groupement d'entreprises chinoises comprend la China Railway Group Limited et la Sinohydro Corporation.

32 Article 1 de la Convention de collaboration du 22 avril 2008.

33 Selon l'article 3.4 de la convention de collaboration, la part du capital du groupement d'entreprises chinoises s'élève à $68 \%$ du capital social contre $32 \%$ pour la RD Congo. 
bien des investissements miniers que des travaux d'infrastructures, parmi les obligations prévues par la convention de collaboration du 22 avril 2008, on distingue celles incombant à la République Démocratique du Congo de celles à charge du groupement d'entreprises chinoises.

Le groupement d'entreprises chinoises s'engagent notamment à mobiliser les investissements nécessaires à la réalisation du Projet minier et d'infrastructures, à tout mettre en œuvre pour réduire le coût de revient, économiser les ressources financières de construction, assurer la moderne conception, la qualité, le haut rendement, la fiabilité et la durabilité des travaux d'infrastructures. ${ }^{34}$

La République Démocratique du Congo s'engage notamment à ce que son entreprise publique GECAMINES cède à la joint-venture minière, les Droits et Titres miniers couvrant des gisements déterminés à l'article 4 de la convention de collaboration du 22 avril 2008; si la joint-venture ne parvient pas à rembourser les investissements et les intérêts des Projets minier et d'Infrastructures dans les 25 ans qui suivent sa création, la République Démocratique du Congo s'engage à rembourser le solde restant par toutes autres voies. ${ }^{35}$

L'engagement de rembourser les investissements et les intérêts des Projets minier et d'infrastructures par toutes autres voies a suscité des vives critiques de la part des partenaires occidentaux, suite aux pressions de ceux-ci, le montant des investissements à été revu à la baisse. C'est sur base des discussions entre la République Démocratique du Congo, le Consortium des entreprises chinoises et le Fonds monétaire international que le projet de financement est passé de 9 milliards de dollars à 6 milliards, dont 3 milliards pour le projet d'exploitation minière et 3 milliards pour le projet d'infrastructures. ${ }^{36}$

La République Démocratique du Congo s'engage également à accorder des avantages commerciaux, fiscaux, douaniers conformément à l'article 14 de la convention de collaboration du 22 avril 2008 ainsi que des garanties contre les risques politiques ainsi que de ne pas appliquer à la joint-venture et à l'entrepreneur chargé des travaux d'infrastructures toute législation ultérieure qui viendrait à porter atteinte aux droits acquis (clause de stabilisation).

\section{Règlement des différends}

L'article 20.1 de la convention de collaboration du 22 avril 2008 prévoit que tout différend relatif à l'interprétation et à l'exécution de la présente convention doit être réglé en priorité par des discussions amiables entre les Parties. L'article 20.2, quant à lui, prévoit une clause compromissoire en disposant que tout différend n'ayant pas été réglé dans les six mois qui suivent les discussions amiables -engagées en vue de résoudre le différend- est soumis à

34 Article 17 de la Convention de collaboration du 22 avril 2008.

35 Article 13.3.4 de la Convention de collaboration du 22 avril 2008.

36 Michel LUNTUMBUE, Le dragon et le léopard : un regard sur les relations sino-congolaises in http://www.cetri.be/spip.php?auteur554, consulté le 29 octobre 2014. 
l'arbitrage du Centre international pour le règlement des différends relatifs aux investissements (CIRDI), selon les règles de celui-ci.

La convention de collaboration en son article 21 prévoit comme droit applicable la loi du lieu de l'acte et stipule qu'au cas où la loi applicable ne peut être déterminée selon le critère précédent, et afin de la déterminer, les parties se réfèreront aux critères usuellement utilisés dans le commerce international pour régler les différends de même nature. La lecture de cet article nous amène à nous interroger sur les raisons qui expliquent le fait que les rédacteurs de la convention n'aient pas été plus précis sur ce qu'ils entendaient par « loi du lieu de l'acte ", une précision qui trouve toute son opportunité dans un contrat contenant un élément d'extranéité capable d'engendrer des difficultés pouvant surgir d'un problème de conflit des lois dans la détermination de la loi applicable. C'est peut-être pour pallier à cette ambigüité qu'il était nécessaire d'ajouter la possibilité de se référer aux critères usuellement utilisés dans le commerce international pour déterminer la loi applicable.

Quoi qu'il arrive, la Convention du CIRDI- à qui cet accord de collaboration reconnait la compétence de régler les différends- stipule en son article 42 que le Tribunal statue sur le différend conformément aux règles de droit adoptées par les parties, faute d'accord entre les partes, le Tribunal applique le droit de l'Etat contractant partie au différend-y compris les règles relatives aux conflits de lois- ainsi que les principes de droit international en la matière. ${ }^{37}$ Conformément à cet article, la CIRDI appliquera le droit congolais et les principes de droit international existant en la matière.

Ceci souligne l'intérêt de déterminer les lois nationales de la RDC applicables au projet minier et au projet d'infrastructures.

IV. Les lois nationales applicables dans la mise en œuvre du projet minier et du projet d'infrastructures mis en place par la convention de collaboration du 22 avril 2008

Parmi les textes de droit commun congolais applicables dans la mise en œuvre du projet minier et du projet d'infrastructures figurent notamment : la Constitution du 18 février 2006, la loi $n^{\circ} 004$ du 21 février 2002 portant Code des investissements, la loi $n^{\circ} 007 / 2002$ du 11 juillet 2002 portant Code minier, la loi $n^{\circ} 015 / 2002$ du 16 octobre 2002 portant Code du travail, les règles régissant le droit des sociétés commerciales qui conformément à l'article 3 de la Convention de Joint-venture du 28 juin 2008 réglementeront la constitution et la gestion de la joint-venture minière ${ }^{38}$.

37 Laurent OKITONEMBO WETSHONGUNDA, « Considérations critiques sur la nature et la portée de la Convention de collaboration signée par la République Démocratique du Congo et un groupe d'entreprises chinoises », in Les Enjeux de la Convention minière RDC-CHINE, Kinshasa, 2010, p.61.

38 La loi n¹0/002 du 11 février 2010 autorisant l'adhésion de la République Démocratique du Congo au traité du 17 octobre 1993 relatif à l'harmonisation du droit des affaires en Afrique (Ohada) permet à l'Acte uniforme relatif au droit des sociétés commerciales et du groupement d'intérêt économique de régir le fonctionnement de la Sicomines. Conformément à l'AUSCGIE actuellement en vigueur en RDC, la Sicomines est une société anonyme. 
Parmi les textes édictés par les autorités congolaises et pris dans la mise en œuvre de la convention du 22 avril 2008, il sied d'évoquer : l'Ordonnance nº8/046 du 14 juillet 2008 autorisant la création d'une société dénommée la Sino-Congolaise des Mines, en sigle «SICOMINES $»^{39}$, le Décret $n^{\circ} 08 / 018$ du 26 août 2008 portant création, organisation et fonctionnement du «Bureau de Coordination et de Suivi du Programme Sino-Congolais » en sigle $« \mathrm{BCSPSC} »^{40}$.

V. Les dérogations au régime de droit commun applicables aux investissements effectués dans le secteur minier

La lecture de certains engagements pris par la RDC au regard des lois nationales applicables aux investissements effectués dans le secteur minier fait apparaître quelques particularités, spécifiques à la convention de collaboration du 22 avril 2008, qui peuvent-être perçues comme des dérogations au régime de droit commun. Les dérogations relatives à l'expropriation et à la clause d'intangibilité et aux exonérations fiscales ont retenu notre attention.

1. Dérogation portant sur le droit à l'expropriation

a) Position du législateur congolais sur le droit à l'expropriation

Parmi les textes qui organisent le droit à l'expropriation de droit interne nous avons notamment :

- La constitution du 18 février 2006 : qui dispose que l'Etat encourage et veille à la sécurité des investissements privés étrangers. Nul ne peut être privé de sa propriété que pour cause d'utilité publique et moyennant une juste et préalable indemnité octroyée selon les conditions fixées par la loi. Nul ne peut être saisi en ses biens qu'en vertu d'une décision prise par une autorité judiciaire compétente. ${ }^{41}$

- Le code des investissements : Conformément à la loi n ${ }^{\circ} 004$ du 21 février 2002 portant code des investissements, l'indemnisation conséquente à l'expropriation est considérée juste si elle est basée sur la valeur de marché de l'actif qui a été nationalisé ou exproprié; cette valeur doit être déterminée d'une manière contradictoire immédiatement avant l'expropriation ou la nationalisation, ou avant que la décision d'exproprier ou nationaliser ne soit devenue du domaine public. ${ }^{42}$

39 Au sens de la convention, la Sicomines est une société de droit congolais et prendra la forme d'une société anonyme telle réglementée par l'Acte uniforme relatif au droit des sociétés commerciales et du groupement d'intérêt économique.

40 Ces textes sont disponibles sur www.congomines.org.

41 Article 34 de la constitution du 18 février 2006.

42 Article 26 de la loi n 004 du 21 février 2002 portant code des investissements. 
- Le Code minier : la loi n007/2002 du 11 juillet 2002 portant Code minier établit comme principe la non-soumission des installations minières ou de carrières à la mesure d'expropriation pour cause d'utilité publique. Cependant si des circonstances exceptionnelles fixées par la loi l'exigent, une indemnité équitable sera versée à la victime au moins six mois avant l'acte matériel d'expropriation. En cas d'abus, la victime peut recourir, selon son choix, au recours arbitral ou judiciaire. ${ }^{43}$

Les dispositions relevées traduisent la volonté de l'Etat congolais d'attirer les investisseurs et de sécuriser les titres qui leurs sont octroyés. Le secteur minier congolais a connu plusieurs crises, au cours de ces dernières décennies dont la première d'ordre structurelle a résulté de la nationalisation des différentes entreprises publiques suite à laquelle elles ont connu dès les années 1970 une mégestion qui est allée en s'accentuant. ${ }^{44}$

b) L'expropriation telle qu'interdite par la convention de collaboration du 22 avril 2008

La convention de collaboration quant à elle, prévoit en son article 15.2 que le gouvernement congolais s'engage à ne jamais et, en aucun cas, s'approprier, nationaliser, ni exproprier directement ou indirectement, la joint-venture minière ainsi que son patrimoine.

De la confrontation des dispositions relatives à la garantie offerte par la RDC au groupement d'entreprises chinoises aux lois nationales régissant les investissements effectués dans le secteur minier, il ressort que contrairement à l'article 34 de la constitution du 18 février 2006, à l'article 26 du code des investissements, ainsi qu'au chapitre 3-Titre X-de la loi n 007 portant code minier, le gouvernement congolais se dépouille de son droit d'expropriation et verrouille, à ses risques et périls, la possibilité que lui offre les lois de la République d'invoquer ce droit pour des raisons exceptionnelles. Si d'aucuns peuvent s'interroger sur la validité de cette stipulation qui parait non conforme aux lois de la République, il est vrai que la RDC ne pourra se prévaloir de toute irrégularité qui en découlerait contre les parties chinoises.

\section{Dérogations relatives à la clause de stabilité}

a) La clause de stabilité telle que réglementée par les lois nationales

L'Etat garantit la stabilité des dispositions constituant le nouveau Code minier et s'interdit de les modifier autrement, si ce n'est que dans la forme prévue dans ce nouveau code. La forme dont il s'agit est indiquée à l'article $276 \mathrm{du}$ Code minier au sujet de la garantie de stabilité en ces termes précis : «l'Etat garantit que les dispositions du présent Code ne peuvent être modifiés que si, et seulement si, le présent Code fait lui-même l'objet d'une modification législative adoptée par le Parlement. Les droits attachés ou découlant d'un

43 Chapitre 3,Titre $\mathrm{X}$ de la loi $\mathrm{n}^{\circ} 007 / 2002$ du 11 juillet 2002 portant Code minier.

44 Lire à ce sujet Optimise Africa, Encyclopédie de l'industrie et du commerce, Kinshasa, 2010, p. 93. 
permis de recherches ou droit minier d'exploitation octroyé et valide à la date de la promulgation d'une telle modification législative ainsi que les droits attachés ou découlant du droit minier d'exploitation octroyé postérieurement en vertu d'un tel permis de recherches incluant, entre autres, les régimes fiscal, douanier et de change du présent Code, demeurent acquis et intangibles pendant une période de dix ans à compter d'une part, de la date de l'entrée en vigueur de la modification législative pour les droits miniers d'exploitation valides existant à cette date, d'autre part de l'octroi du droit minier d'exploitation octroyé postérieurement en vertu d'un Permis de Recherches valide existant à la date de l'entrée en vigueur de la modification législative ».

b) La Clause de stabilité telle que formulée par la convention de collaboration du 22 avril 2008

L'Article 14.4 dispose que sans préjudice des avantages acquis dans le cadre de la présente convention de collaboration, la joint-venture minière et l'entrepreneur chargé des travaux d'infrastructures bénéficieront des avantages résultant de toutes les nouvelles dispositions légales et réglementaires qui seraient ultérieurement prises par la RDC ou des conventions que celle-ci viendrait à conclure avec d'autres investisseurs. Néanmoins, toutes nouvelles dispositions légales et réglementaires apportant des désavantages à leur égard ne leur seront pas appliquées.

Force est de constater que les garanties juridiques accordées au groupement des entreprises chinoises semblent être plus étendues que celles prévues par les lois de la République. Constatant cet état des choses, le gouvernement qui est le récipiendaire des moyens financiers qui découleront de ce projet devra mettre en place les conditions préalables destinées à doter l'Etat d'infrastructures dont la population congolaise a besoin et de relancer le secteur minier, créateur d'emplois et de richesses.

Cette clause de stabilité est une preuve que le développement du projet minier et d'infrastructures déroge au droit commun applicable aux activités du secteur minier développées en République Démocratique du Congo.

\section{Les dérogations relatives aux exonérations fiscales}

a) Position du législateur congolais sur la fiscalité des investissements dans le secteur minier

Le code minier exprime la politique fiscale que le législateur met en œuvre en ces termes $^{45}$ : Les conventions minières ont eu pour conséquence l'amenuisement des recettes du Trésor public à cause de la généralisation des exonérations. La fiscalité étant pour les entreprises minières un des facteurs déterminants de la décision d'investir leurs capitaux dans un pays donné, le présent Code introduit une innovation en mettant en place un régime 
fiscal et douanier unique applicable à tous les opérateurs du secteur minier industriel et à tous les exploitants miniers à petite échelle, sans aucune exception, résultant de la nature ou de la durée du titre minier. Dans le but de maximiser les recettes de l'Etat, le régime fiscal et douanier du Code minier est dominé par le principe de la non-exonération. Contrairement aux avantages fiscaux consentis dans les conventions minières qui allaient jusqu'à accorder des exonérations durant des années au préjudice du Trésor Public, ceux accordés par le présent Code se limitent principalement au rabattement du taux de la contribution. Il en résulte que la caisse du trésor est désintéressée à n'importe quelle phase de l'investissement minier.

La position du législateur peut-être comprise et s'explique par le fait que la manne fiscale dans le domaine est encore faible : les entreprises du secteur auraient versé à l'Etat 83 millions de dollars de droits et impôts divers en 2007 d'après le rapport du conciliateur de l'Initiative pour la Transparence des Industries Extractives (ITIE, 2007). ${ }^{46}$ La même année, le Katanga a exporté 1,5 milliard de dollars américains de minerais pour 15 millions de taxe tandis que le Kasaï occidental a exporté 1 milliard de dollars américains de diamant pour 40 millions de dollars allant à l'Etat ${ }^{47}$. Cette faible rentabilité s'explique par les exonérations consenties pour accueillir des investissements massifs dans le secteur mais aussi, malgré le renforcement des contrôles par les autorités, par les nombreuses filières d' " évasion minière ${ }^{48}{ }^{48} \mathrm{Il}$ est possible d'améliorer le rendement de l'impôt dans le secteur minier et ce, sans alourdir la pression fiscale notamment, à travers une meilleure surveillance des exportations de minerais dans les zones sortant parfois de près d'une décennie de conflits armées. $^{49}$

b) Les exonérations fiscales contenues dans la convention de collaboration

Contrairement au Code minier, la convention de collaboration, elle, prévoit que la RDC concède, pendant les périodes de remboursement de l'investissement minier et de l'investissement de la première tranche d'infrastructures, les avantages ci-après ${ }^{50}$ :

- l'exonération totale de tous les impôts, droits, taxes, douanes et redevances, directs ou indirects, à l'intérieur ou à l'import et l'export, payables en RDC et ceux liés aux activités minières et au développement d'infrastructures. ${ }^{51}$

- l'exemption des frais et paiement relatifs à la demande, à l'octroi, au transfert et à la cession des Droits et Titres miniers, des permis de prospection et d'exploitation. ${ }^{52}$

46 Source citée par Optimise Africa, Encyclopédie de l'Industrie et du Commerce, Kinshasa, Optimise Africa, 2010, p. 94.

47 Idem.

48 Optimise Africa, op.cit, Kinshasa, 2010, p. 94.

49 Optimise Africa, op.cit, Kinshasa, p.94.

50 Article 14.2 de la Convention de collaboration du 22 avril 2008.

51 Article 14.2.1 de la Convention de collaboration du 22 avril 2008.

52 Article 14.2.2 de la Convention de collaboration du 22 avril 2008. 
Nous présumons que la particularité des avantages accordés par la RDC découle de l'espoir que la convention de collaboration lui serve d'outil de promotion des droits économiques et sociaux des populations congolaises et de l'amélioration des conditions de vie des populations congolaises.

\section{B. Les incidences de la convention de collaboration du 22 avril 2008 sur les droits économiques et sociaux des populations congolaises}

I. Fondements juridiques des droits économiques et sociaux reconnus aux populations congolaises

Plusieurs textes juridiques existant aussi bien niveau international que national consacrent et concourent à la protection des droits économiques et sociaux des populations congolaises.

\section{Protection internationale des droits économiques et sociaux}

En réalité, depuis l'adoption de la Déclaration universelle des droits de l'homme en 1948, le droit international reconnaît pleinement les droits économiques, sociaux et culturels. ${ }^{53}$

Plusieurs instruments internationaux ratifiés par la RDC et la Chine visent à protéger et à promouvoir les droits économiques et sociaux des populations congolaises. Il s'agit en l'occurrence du Pacte international relatifs aux droits économiques, sociaux et culturels et la Convention relative aux droits de l'enfant ${ }^{54}$ - dont les dispositions doivent être respectées par toutes les entreprises qui opèrent dans le cadre de l'accord sino-congolais.

En outre, la RDC est signataire de la charte africaine des droits de l'homme et des peuples qui va bien au-delà d'une simple mention des droits économiques, sociaux et culturels; elle les place juridiquement sur un pied d'égalité avec les droits civils et politiques et leur confère même un caractère contraignant. ${ }^{55} \mathrm{Le}$ gouvernement chinois a ratifié quatre des huit conventions fondamentales de l'Organisation internationale du Travail, dont celles concernant l'égalité de rémunération, la discrimination et les pires formes de travail des enfants. ${ }^{56}$

53 Kofi Kumado, "La surveillance des droits économiques, sociaux et culturels ", Contribution à la Conférence de la Commission internationale des juristes (CIJ) sur les Droits économiques, sociaux et culturels et le rôle des juristes, tenue à Bangalore, Inde, du 23 au 25 octobre 1995.in Droits économiques, sociaux et culturels et le rôle des juristes, $55^{\text {ème }}$ numéro de la Revue de la Commission internationale des juristes, Genève, 2005, p111.

54 Voir Collection des traités des Nations Unies, source citée par Global Witness, La Chine et le Congo : Des amis dans le besoin, p. 37.

55 Kofi Kumado, op.cit, pp111, note 51.

56 Rights and Accountability in Development, " Chinese mining operations in Katanga, Democratic Republic of Congo », September 2009 source citée par Global Witness, La Chine et le Congo : Des amisdans le besoin, p. 37. 
2. Protection nationale des droits économiques et sociaux des populations congolaises

La constitution de la RDC dispose que 1' Etat garantit le droit au travail, la protection contre le chômage et une rémunération équitable et satisfaisante assurant au travailleur ainsi qu'à sa famille une existence conforme à la dignité humaine, complétée par tous les autres moyens de protection sociale, notamment la pension de retraite et la rente viagère (article 36 de la constitution), la liberté syndicale est reconnue et garantie (article 38), le droit de grève est reconnu et garanti (article 39), toute personne a droit à l'éducation scolaire (article 43), le droit à la santé et à la sécurité alimentaire est garanti (article 47), le droit à un logement décent, le droit à l'accès à l'eau potable, et à l'énergie électrique sont garantis (article 48).

II. La Convention minière de 2008 comme outil de promotion et de protection des droits économiques et sociaux: Bilan et recommandations pour un usage efficient des financements issus des investissements chinois

Cette convention est un outil permettant à l'Etat de mettre en œuvre sa politique économique et de pourvoir aux droit économiques et sociaux que la constitution reconnait aux congolaises et aux congolais 'dans la mesure où elle contient les projets d'infrastructures ${ }^{57}$ qui peuvent contribuer à améliorer les conditions économiques et sociales des populations congolaises. A titre d'exemple, la construction des routes telle que prévue par la convention de collaboration pourrait faciliter l'écoulement des marchandises, la réduction des coûts de transports et par conséquent celle des denrées alimentaires et permettre à l'Etat de pourvoir à la sécurité alimentaire des populations congolaises, qui est consacrée comme droit fondamental. $^{58}$

Bien que la convention de collaboration n'énumère pas expressis verbis les droits économiques et sociaux qu'elle promeut, elle contient néanmoins des dispositions qui impliquent la construction d'infrastructures pouvant avoir un effet positif dans le développement de l'Etat et par conséquent dans l'amélioration des conditions économiques et sociales des populations congolaises.

\section{Bilan}

Le groupement d'entreprises chinoises s'est engagé à mobiliser 6 milliards USD pour le financement du projet d'infrastructures, ainsi que 3,2 milliards USD pour financer le Projet

57 La liste des projets d'infrastructures figure à l'annexe C de la convention du 22 avril 2008 et comprend : la construction des chemins de fer, de routes bitumées, la réhabilitation des routes, des aéroports, construction et équipements des hôpitaux, construction de barrages hydro-électriques, construction et réhabilitation des centre de formation, construction des logements sociaux, des centres de santé et d'universités.

58 Article 47 de la constitution du 18 février 2006. 
Minier. ${ }^{59}$ Sous la pression des institutions financières internationales à savoir le Fonds Monétaire International et la Banque Mondiale, le volet construction s'est amoindri de moitié pour se situer à 3 milliards USD. ${ }^{60}$

Lors du petit déjeuner de presse organisé à l'occasion des 5 ans que totalisait la convention de collaboration du 22 avril 2008, le Secrétaire Exécutif du Bureau de Coordination et de Suivi du Programme Sino-Congolais ${ }^{61}$ affirme que depuis la signature de la Convention et malgré les écueils rencontrés, le Projet de Coopération Sino-Congolais a déjà engrangé plusieurs réalisations, essentiellement au volet du développement des infrastructures publiques et communautaires ${ }^{62}$ et renseigne que ${ }^{63}$ :

- avec le prêt de USD 350 millions obtenu en janvier 2009 d'EXIM BANK, la République Démocratique du Congo a financé la première tranche des travaux d'infrastructures, notamment : le Boulevard du 30 juin (Lot 1), l'Avenue du tourisme, la Bretelle de Lutendele, le tronçon Lubumbashi-Kasomeno sur la RN5, le tronçon routier Beni-Luna sur la RN4 et l'hôpital du cinquantenaire;

- avec le prêt d'Exim Bank de USD 118 millions, le Congo a pu financer la deuxième tranche des travaux d'infrastructures à savoir : Boulevard du 30 juin (Lot 2), Boulevard Triomphal et Sendwe, Esplanade du Palais du Peuple, l'acquisition d'une unité de production des préfabriqués pour le volet habitat, l'acquisition des groupes électrogènes pour accroitre le taux de desserte en électricité de certains chefs-lieux des provinces et l'acquisition des poteaux solaires pour l'éclairage public de certains chefs-lieux des territoires;

- avec le montant de USD 328 millions, apportés en août 2012 comme participation de la partie congolaise $(50 \%)$ au développement du projet, la République Démocratique du Congo est entrain de financer les travaux de construction en cours de la centrale hydro-

59 Bilan dressée lors du petit déjeuner de presse organisé par le Secrétaire Exécutif du Bureau de Coordination et de Suivi du Programme Sino-Congolais mis en ligne le 25 mai 2013 sur http://www.1 econgolais.cd/contrats-chinois-cinq-ans-deja/ consulté le 28 octobre 2014.

60 Idem.

61 L'article 3 du Décret nº8/018 du 26 août 2008 portant création, organisation, fonctionnement du Bureau de Coordination et de Suivi du Programme Sino-Congolais en sigle « B.C.S.P.S.C » stipule qu'il a pour objet la gestion et le suivi de la mise en œuvre des accords de coopération signés entre le Gouvernement de la République Démocratique du Congo et les partenaires privés chinois dans les domaines minier et des infrastructures de base. Ce texte est disponible sur le site : http : www.congomines.org.

62 Bilan du programme Sino-Congolais dressé par le Secrétaire exécutif du Bureau de Coordination et de Suivi du Programme Sino-Congolais lors du petit déjeuner de presse organisé à l'occasion des cinq ans que totalise le Projet de coopération BCPSC-2013-Bilan-5ans-Programme-SinoCongolais.pdf disponible sur http://www.congomines.org/fr/bcpsc-2013-bilan-5-ans-du-projet-sino -congolais/ consulté le 29 octobre 2014.Le secrétaire exécutif renseigne qu'il a été prévu un décaissement d'1,5 milliard de dollar américain en faveur des projets d'infrastructures avant la mise en production du Projet Minier (Sicomines).

63 La situation complète des décaissements et des infrastructures réalisées est disponible sur le site précédemment cité. 
électrique de Busanga. Les discussions sont en cours avec les entreprises chinoises pour trouver un mécanisme de financement du projet Busanga en BOT et dégager ainsi le montant de USD 328 millions en faveur des projets d'infrastructures à réaliser avant la mise en production de la Sicomines, c'est-à-dire avant fin 2015.

Les réalisations énumérées font de la convention du 22 avril 2008, un outil de promotion de la sécurité alimentaire, de la santé, et de l'électricité qui sont des droits économiques et sociaux reconnus aux populations congolaises mais ne mettent pas les relations sino-congolaises à l'abri des critiques formulées à son sujet dont celle relative au fait pour la Chine de ne pas subordonner son aide au respect de la démocratie et des droits de l'homme $^{64}$ et de ne pas mettre en avant la conditionnalité politique comme critère principal pour enclencher toute forme de coopération ${ }^{65}$.

2. Conditions préalables et recommandations pour un usage efficient des investissements chinois

Le diagnostic qualitatif et quantitatif a établi sans équivoque que les congolais vivent la pauvreté à travers des dimensions tangibles (la détérioration des infrastructures de transport et de communication, les besoins fondamentaux non satisfaits, la détérioration des facteurs de production, le faible accès aux services sociaux de base) et intangible (l'absence de paix, la culture d'impunité renforçant la corruption, l'injustice) ${ }^{66}$. Cette situation explique la volonté du gouvernement congolais de trouver les ressources financières nécessaires à la réalisation des projets d'infrastructures nationales estimées importantes et urgentes ${ }^{67}$ et de mettre en œuvre une politique censée répondre aux besoins en infrastructures, en eau, en électricité, de santé et éducation emploi, logement. ${ }^{68}$

Pour y parvenir les conditions préalables à l'assainissement du milieu des affaires doivent être prises en compte dans la mise en œuvre de la convention du 22 avril 2008, aussi bien dans le développement du projet d'infrastructures que dans celui du projet minier.

Pour ce faire, il conviendrait d'abord de :

- Lutter contre la corruption et promouvoir la bonne gouvernance. La corruption peut être entendue comme un usage abusif d'une charge publique aux fins de profit personnel et peut engendrer des contrats léonins, la fraude fiscale, les détournements des deniers pu-

64 Voir Rigobert MINANI BIHUZO, op.cit, p.32, note 5..

65 Adama Gaye, op.cit, p.248, note 23.

66 Ministère du plan de la République démocratique du Congo, Combattre la pauvreté en RDC : Gouvernance et initiatives de la population -Document stratégique de croissance et de réduction de la pauvreté en bref, 2007, p.4.

67 Objectif de l'Etat congolais tel que repris dans l'article 1 de la Convention de collaboration du 22 avril 2008.

68 Voir les axes prioritaires de la politique gouvernementale tels qu'indiqués par le Secrétaire Exécutif du Bureau de Coordination et de Suivi du Programme Sino-Congolais, in http://www.congomin es.org/fr/bcpsc-2013-bilan-5-ans-du-projet-sino-congolais/ consulté le 29 octobre 2014. 
blics sous forme de surfacturation des commandes publiques. ${ }^{69}$ Dans le cas de la République Démocratique du Congo, le contrôle de la corruption peut s'accompagner d'un taux de croissance économique supplémentaire de l'ordre de $8 \%$ l'an, soit d'un taux de croissance de PIB par habitant de l'ordre de 5\%. ${ }^{70}$

Il est aujourd'hui prouvé qu'un pays qui pratique la bonne gouvernance au niveau politique, administratif et économique est capable de réaliser les objectifs d'un développement durable et de positionner l'ensemble de la Nation sur un sentier porteur d'espoir et de bienêtre. $^{71}$

- Respecter les conventions internationales et les lois nationales protégeant les droits des travailleurs

Les gouvernements chinois et congolais ont tous les deux ratifié des normes internationales dans le domaine des droits de l'homme, notamment le Pacte international relatifs aux droits économiques, sociaux et culturels et la Convention relative aux droits de l'enfant- dont les dispositions doivent être respectées par toutes les entreprises dans le cadre de l'accord sinocongolais. ${ }^{72}$ La RDC devra également veiller au respect des conditions d'hygiène et de sécurité prévues par l'article 187 de la loi n $015 / 2002$ portant code du travail.

- Promouvoir la transparence dans la gestion des financements reçus. La transparence est un élément essentiel à l'instauration de la bonne gouvernance. ${ }^{73}$

C'est ainsi que l'Initiative pour la Transparence dans les Industries Extractives (ITIE) avait adressé une note à la République Démocratique du Congo pour l'inclusion du partenariat congolais « Sicomines » dans le référentiel des paiements et revenus à déclarer.

L'ITIE a salué les efforts accomplis par la RDC et donc déclaré le 02 juillet 2014, l'Etat congolais conforme aux règles de l'ITIE. ${ }^{74} \mathrm{Il}$ est donc important de continuer sur cette lancée.

Nous pensons qu'il faudrait également :

- Associer la société civile au suivi effectué par le Bureau de coordination de suivi du programme sino-congolais et veiller au respect des zones interdites et des matières réservées.

69 Antoine BATAMBA BALEMBU, « Corruption, gouvernance et perspectives des Objectifs du Millénaire pour Développement » in Contribution à la réalisation des Objectifs du Millénaire pour le Développement en République démocratique du Congo, Kinshasa, 2012, p. 164.

70 Antoine BATAMBA BALEMBU, op.cit, p.165, note 69.

71 Idem.

72 Collection des traités des Nations Unies, http : //treaties.un.org/, source citée par Global Witness, La Chine et le Congo : Des amis dans le besoin, Un rapport de Global Witness sur la RDC, Mars 2011, p.37.

73 Lire Abdeslam EL HAROUCHY, « Réflexions sur la problématique de la lutte contre la vulnérabilité à la corruption » in Contribution à la réalisation des objectifs du millénaire pour le développement en République démocratique du Congo, (Auteur : Institut des Recherches Economiques et Sociale -IRES-, Kinshasa, 2012, p.137.

74 Voir ITIE, http://eiti.org/fr/republique-democratique-du-coffngo consulté le 25 octobre 2014. 
- Soustraire de la clause de stabilité le pouvoir, accordé par le Code minier au Président de la République, de déclarer une zone interdite aux activités minières ou aux travaux de carrière lorsque la sûreté nationale, la sécurité des populations, l'incompatibilité de l'activité minière et des travaux de carrières avec d'autres usages existants ou planifiés du sol ou du sous-sol ainsi que la protection de l'environnement l'exigent. ${ }^{75}$

- Publier les montants alloués à la rémunération des travailleurs qui participent à la construction des infrastructures prévus à l'annexe C de la convention de 2008.

- Faciliter l'accès des populations locales aux centres de santé, écoles, hôpitaux et autres services sociaux qui seront construits dans le cadre du projet et pourvoir un service de qualité en les dotant d'un personnel qualifié et bien rémunéré.

- Veiller à ce que les coûts de ces services soit accessibles aux différentes couches de la population et indiquer les sources de financement nécessaire au fonctionnement des services construits et à la rémunération du personnel qui y travaille.

- Assurer une coordination entre le Bureau de Coordination et de Suivi du Programme Sino-Congolais et le service de protection de l'environnement minier.

\section{CONCLUSION}

Le cadre juridique des relations économiques et commerciales entre la RDC et la Chine peut être appréhendé sous deux volets. Le premier qui porte sur les rapports interétatiques tels que réglementés notamment par les principes et règles de droit international et le second sur les rapports transnationaux marqués singulièrement par la convention de collaboration du 22 avril 2008 relative au développement d'un projet minier et d'un projet d'infrastructures en RDC qui, s'inscrivant dans la logique de la politique économique de la Chine telle que conçue avec ses partenaires africains dans le cadre du Forum Chine-Afrique, a donné lieu à l'exploitation des ressources naturelles en échange d'un financement des travaux d'infrastructures dont la RDC a besoin en vue de l'amélioration des conditions de vie des populations congolaises.

Bien que cette convention ait permis la réalisation de plusieurs infrastructures, elle n'a pas encore pleinement répondu aux attentes des populations congolaises. Ceci s'explique en partie par le fait que les financements prévus pour la construction des infrastructures n'ont pas encore été totalement décaissés et que le Projet minier que symbolise la Sicomines, n'a pas encore amélioré les recettes du secteur minier d'autant plus la première tonne de cuivre produite par la Sicomines est attendue en $2015^{76}$.

Pour que la convention remplisse pleinement les objectifs que les parties lui assignent et serve d'outil de promotion et de protection des droits économiques et sociaux des popu-

75 Voir Chapitre premier du Titre premier sur les généralités de la loi nº07/2002 du 11 juillet 2002 portant Code minier.

76 Bilan dressée lors du petit déjeuner de presse organisé par le Secrétaire Exécutif du Bureau de Coordination et de Suivi du Programme Sino-Congolais mis en ligne le 25 mai 2013 sur http://www.1 econgolais.cd/contrats-chinois-cinq-ans-deja/ consulté le 28 octobre 2014. 
lations congolaises, l'Etat congolais devra mettre en place un cadre propice à l'émergence des principes de bonne gouvernance, de gestion transparente qui permettront d'affecter, comme il se doit, les financements issus de l'exploitation des ressources naturelles à l'amélioration des conditions de vie des populations congolaises.

\section{BIBLIOGRAPHIE}

1. Adama Gaye, Le dragon et l'autruche. Essai d'analyse de l'évolution contrastée des relations sino-africaines : sainte ou impie alliance du XXI ${ }^{\mathrm{ème}}$ siècle, Paris, L'Harmattan, 2006.

2. Alain Frachon, «Dix ans de la Chine à l'OMC : bilan » in journal Le Monde, chronique internationale du 29 septembre 2011 disponible sur http//www.LeMonde.fr, consulté le 07octobre 2014.

3. BAKANDEJA WA MPUNGU Grégoire BakandejawaMpungu, Le droit commercial international: Les Peurs justifiées de l'Afrique face à la mondialisation des marchés, Kinshasa, Paris, Bruxelles, Afrique éditions, De Boeck et Larcier, 2001.

4. BATAMBA BALEMBU Antoine, « Corruption, gouvernance et perspectives des Objectifs du Millénaire pour Développement » in Contribution à la réalisation des Objectifs du Millénaire pour le Développement en République démocratique du Congo, Kinshasa, Editions Universitaires Africaines, 2012, pp 163-204.

5. BLACKHURST Richard, « Renforcement des capacités en Afrique pour une participation efficace au processus de l'OMC », in L'Afrique et les défis de l'OMC, Paris, Ed. KARTHALA, 2004, pp1-29.

6. CHARPENTIER Jean, Institutions internationales, Paris, Dalloz, 2002.

7. Convention de collaboration du 22 avril 2008 disponible sur http://www.congomines.o $\mathrm{rg} / \mathrm{fr} /$ convention-de-collaboration-sino-congolaise-groupement-dentreprises-chinoisesrdc-2008/

8. EL HAROUCHY Abdeslam, « Réflexions sur la problématique de la lutte contre la vulnérabilité à la corruption » in Contribution à la réalisation des objectifs du millénaire pour le développement en République démocratique du Congo, Institut des Recherches Economiques et Sociale, Kinshasa, Editions Universitaires Africaines, Kinshasa, 2012, pp.135-141.

9. Global Witness, La Chine et le Congo : Des amis dans le besoin, Rapport sur la République démocratique du Congo, Mars 2011.

10. http : //cd.china-embassy.org/fra/xw/more/t215218.htm consulté le 28 octobre 2014.

11. http://eiti.org/fr/republique-democratique-du-congo consulté le 25 octobre 2014.

12. http://www.congomines.org/fr/convention-de-collaboration-sino-congolaise-groupeme nt-dentreprises-chinoises-rdc-2008/ consulté le 28 septembre 2014.

13. http://www.globalwitness.org/fr/library/contrat-sino-congolais-de-nouveaux-document s-publi\%C3\%A9S mis en ligne le 1 1 er août 2011 et consulté le 10 août 2014.

14. http://www.lecongolais.cd/contrats-chinois-cinq-ans-deja/ consulté le 28 octobre 2014. 
15. http://www.wto.org/french/thewto_f/whatis_f/org6_f.htm consulté le 15 octobre 2014.

16. Kumado Kofi, « La surveillance des droits économiques, sociaux et culturels », Contribution à la Conférence de la Commission internationale des juristes (CIJ) sur les Droits économiques, sociaux et culturels et le rôle des juristes, tenue à Bangalore, Inde, du 23 au 25 octobre 1995.« La surveillance des droits économiques, sociaux et culturels » in Droits économiques, sociaux et culturels et le rôle des juristes, $55^{\text {ème }}$ numéro de la Revue de la Commission internationale des juristes, Genève, 2005.

17. KUMBU ki NGIMBI, Législation en matière économique, Kinshasa, Gallimage, 2013.

18. KUMBU ki NGIMBI, Protection légale des investissements privés étrangers en République Démocratique du Congo et coopération eurafricaine, Thèse de Doctorat, Hambourg, RFA, 1998.

19. Loi $n^{\circ}$ 007/2002 du 11 juillet 2002 portant Code minier, Journal Officiel, numéro spécial du 15 juillet 2002.

20. Loi nº04-2002 du 21 février 2002 portant Code des investissements, in Codes Larciers, T III, vol.2.

21. Loi $n^{\circ} 015 / 2002$ du 16 octobre 2002 portant Code du travail, Journal Officiel de la RD Congo, $43^{\text {ème }}$ année, numéro spécial du 25 octobre 2002.

22. Loi n $10 / 002$ du 11 février 2010 autorisant l'adhésion de la RD Congo au traité du 17 octobre 1993 relatif à l'harmonisation du droit des affaires en Afrique, Journal Officiel de la RD Congo, $51^{\text {ème }}$ année, Kinshasa, 3 mars 2010.

23. LUKE David F., «Renforcement des capacités dans le domaine du commerce pour une meilleure participation de l'Afrique au sein de l'économie mondiale, in L'Afrique et les défis de l'OMC, Paris, Ed. Karthala, 2004, pp 29-47.

24. LUNTUMBUE Michel, Le dragon et le léopard : un regard sur les relations sinocongolaises http://www.cetri.be/spip.php?auteur554.pdf, consulté le 29 octobre 2014.

25. MINANI BIHUZO Rigobert, «La Coopération Chine-Afrique et le débat sur les contrats chinois en RDC» in Les Enjeux de la Convention minière RDC-CHINE, Kinshasa, CEPAS, 2010, pp.23-38.

26. Ministère du plan de la République démocratique du Congo, Combattre la pauvreté en RDC : Gouvernance et initiatives de la population -Document stratégique de croissance et de réduction de la pauvreté en bref, 2007.

27. OKITONEMBO WETSHONGUNDA Laurent, « Considérations critiques sur la nature et la portée de la Convention de collaboration signée par la République Démocratique du Congo et un groupe d'entreprises chinoises ", in Les Enjeux de la Convention minière RDC-CHINE, Kinshasa, CEPAS, 2010, pp 39-64.

28. Optimise Africa, Encyclopédie de l'Industrie et du Commerce, Kinshasa, 2010.

29. RAINELLI Michel, L'Organisation mondiale du commerce, Paris, La Découverte, 2002. 


\section{Impressum}

\section{KAS African Law Study Library - Librairie Africaine d'Etudes Juridiques}

Herausgeber: Konrad-Adenauer-Stiftung e.V., Klingelhöferstraße 23, 10785 Berlin

Schriftleitung: RA Prof. Dr. Hartmut Hamann, Falkertstraße 82, D-70193 Stuttgart Telefon: +49 (o) 711 120950-30 | Fax : +49 (o) 711 120950-50

Mail: hamann@hamann-legal.de

\section{Erscheinungsweise: 4 Ausgaben pro Jahr}

Druck und Verlag: Nomos Verlagsgesellschaft mbH \& Co. KG - Waldseestr. 3-5 D-76530 Baden-Baden. Telefon +49-7221-2104-0 / Fax 49-7221-2104-27 E-Mail:nomos@nomos.de

Anzeigen: sales friendly Verlagsdienstleistungen - Inh. Frau Bettina Roos - Pfaffenweg 15 - D-53227 Bonn Telefon +49-228-97898-o / Fax +49-228-97898-20 E-Mail: roos@sales-friendly.de

Urheber- und Verlagsrechte: Die Zeitschrift und alle in ihr enthaltenen einzelnen Beiträge sind urheberrechtlich geschützt. Jede Verwertung außerhalb der engen Grenzen des Urheberrechtsgesetzes ist ohne Zustimmung des Verlages unzulässig. Das gilt insbesondere für Vervielfältigungen, Übersetzungen, Mikroverfilmungen und die Einspeicherung und Verarbeitung in elektronischen Systemen. Der Nomos Verlag beachtet die Regeln des Börsenvereins des Deutschen Buchhandels e.V. zur Verwendung von Buchrezensionen.

ISSN 2363-6262

\section{Nomos}

